\section{LNG may still be the most cost-effective oral emergency contraception method}

It was with great interest that we read the economic analysis of Thomas et al. ${ }^{1}$ We know now if several assumptions are made, oral emergency contraception (EC) with ulipristal acetate (UPA) is the cheapest option for the health service. The assumptions implicit to the analysis were:

- Meta-analysis of two underpowered randomised controlled trials (RCTs) ${ }^{2}$ using slightly different drug regimes is an acceptable method to determine the effectiveness of UPA and levonorgestrel (LNG) in the real world.

- If pregnancy occurs following UPA exposure, outcomes are the same as for that observed in two RCTs following UPA or LNG exposure.

- Pregnancy following exposure to UPA will incur average costs.
- Neonatal costs do not differ between LNG and UPA and can thus be ignored.

- Unprotected sex does not occur after the administration of EC.

While a discussion of Assumption 1 is beyond the scope of this letter, we would like to address Assumptions 2-5 in more detail.

\section{Assumption 2}

No large study of pregnancy outcomes after exposure to UPA has been published to date. However, the action of UPA on the endometrium and myometrium could at least potentially affect placentation.

The muscle tone of the uterine isthmus is under the control of progesterone and estrogen. ${ }^{3}$ Early luteal phase administration of UPA could reduce the effect of progesterone on the myometrium and could result in a widening of the lower segment of the uterus and a subsequently increased risk of placenta previa.

It is not known if UPA exposure at implantation affects the process of trophobast invasion. It is, however, known that mifepristone administered in the early luteal phase affects the products of at least 28 genes that are likely to regulate implantation ${ }^{4}$ and possibly trophobast invasion. Deficient placentation could increase the risk of pre-eclampsia, intrauterine growth restriction, placental abruption, morbidly adherent placenta and premature delivery. While we accept that this assumption is not supported by strong evidence, it is likely that it will influence obstetrician behaviour (see below).

\section{Assumption 3}

In the absence of reassuring data we expect that obstetricians will decide to offer more than just routine care to women who took a 'black triangle drug' that might cause placental problems. An additional visit here, an extra scan there - even in the absence of any pathology detected, costs would soon add up.

\section{Assumption 4}

No large study of pregnancy outcomes after exposure to UPA has been published so far. However, placental problems could be increased in pregnancies exposed to UPA at the time of implantation then it is possible that neonatal costs in UPA exposed pregnancies will wipe out any potential cost savings.

\section{Assumption 5}

Economic analysis is a commissioning tool that should inform decisions at population level. There is no evidence that better access to EC and increased 
uptake of EC reduce pregnancy rates. ${ }^{56}$ The most likely explanation for this is that EC does not prevent pregnancies from future unprotected sex. If EC does not affect pregnancy rates at population level then the more expensive but ultimately equally ineffective method cannot be more cost effective than a less expensive method.

Although we do not know if early luteal phase UPA exposure causes placental problems is it reasonable to assume that the obstetrician's response will be to 'err on the side of caution', an action that usually incurs a higher level of costs.

We believe that at least Assumptions 2-5 are currently not supported by published evidence and that it is currently far too early an economic analysis.

At present LNG should still be seen as the most cost effective oral EC method available. The real EC question is however not "should we use UPA or LNG" but how can we improve the acceptability and provision of emergency IUDs.

Rudger Pittrof, MSc, MRCOG

Consultant, Enfield Community Services, RASH, London, UK,rudiger.pittrof@enfield.nhs.uk

Ulrike Sauer, DRCOG

Enfield Community Services, RASH, London, UK; ulrike.sauer@enfield.nhs.uk

\section{Palwasha Ikram, MBBS}

Enfield Community Services, RASH, London, UK; Palwasha.lkram@enfield.nhs.uk

Competing interests None.

J Fam Plann Reprod Health Care 2011;37:121-122. doi:10.1136/jprhc.2011.0070

\section{REFERENCES}

1 Thomas CM, Schmid R, Cameron S. Is it worth paying more for emergency hormonal contraception? The cost-effectiveness of ulipristal acetate versus levonorgestrel $1.5 \mathrm{mg}$. J Fam Plann Reprod Health Care 2010:36:197-201.

2 Glasier AF, Cameron ST, Fine PM, et al. Ulipristal acetate versus levonorgestrel for emergency contraception: a randomised non-inferiority trial and meta-analysis. Lancet 2010;375:555-562.

3 Mueller A, Siemer J, Schreiner S, et al. Role of estrogen and progesterone in the regulation of uterine peristalsis: results from perfused non-pregnant swine uteri. Hum Reprod 2006:21:1863-1868.

4 Ghosh D, Sharkey AM, Charnock-Jones DS, et al. Effect of low-dose mifepristone administration on day 2 after ovulation on transcript profiles in implantation-stage endometrium of rhesus monkeys. Reproduction 2009; 138:357-370.

5 Polis CB, Grimes DA, Schaffer K, et al. Advance provision of emergency contraception for pregnancy prevention. Cochrane Database Syst Rev 2007;2:CD005497.

6 Raine TR, Harper CC, Rocca CH, et al. Direct access to emergency contraception through pharmacies and effect on unintended pregnancy and STIs: a randomized controlled trial. JAMA 2005;293:54-62. 